\title{
(2) OPEN ACCESS \\ International Olympic Committee (IOC) Sport Mental Health Assessment Tool 1 (SMHAT-1) and Sport Mental Health Recognition Tool 1 (SMHRT-1): towards better support of athletes' mental health
}

\author{
Vincent Gouttebarge (1),${ }^{1,2}$ Abhinav Bindra, ${ }^{3}$ Cheri Blauwet, ${ }^{4}$ Niccolo Campriani, ${ }^{5}$ \\ Alan Currie (i) ${ }^{6,7}$ Lars Engebretsen, ${ }^{8,9}$ Brian Hainline (1) , ${ }^{10}$ Emily Kroshus, ${ }^{11,12}$ \\ David McDuff $\left(1,{ }^{13}\right.$ Margo Mountjoy $\left(10,{ }^{14}\right.$ Rosemary Purcell, ${ }^{15,16}$ \\ Margot Putukian $\odot{ }^{17}$ Claudia L Reardon, ${ }^{18}$ Simon M Rice, ${ }^{15,16}$ Richard Budgett ${ }^{9}$
}

\begin{abstract}
- Additional material is published online only. To view, please visit the journal online (http://dx.doi.org/10.1136/ bjsports-2020-102411).
\end{abstract}

For numbered affiliations see end of article.

Correspondence to Dr Vincent Gouttebarge, Amsterdam UMC, Univ of Amsterdam, Department of Orthopaedic Surgery, Amsterdam, Netherlands; v.gouttebarge@amsterdamumc. $\mathrm{nl}$

Accepted 18 August 2020 Published Online First 18 September 2020
Check for updates

(C) Author(s) (or their employer(s)) 2021. Re-use permitted under CC BY-NC. No commercial re-use. See rights and permissions. Published by BMJ.

To cite: Gouttebarge $V_{\text {, }}$

Bindra A, Blauwet $C$,

et al. Br J Sports Med

2021:55:30-37

\section{ABSTRACT}

Objectives To develop an assessment and recognition tool to identify elite athletes at risk for mental health symptoms and disorders.

Methods We conducted narrative and systematic reviews about mental health symptoms and disorders in active and former elite athletes. The views of active and former elite athletes $(\mathrm{N}=360)$ on mental health symptoms in elite sports were retrieved through an electronic questionnaire. Our group identified the objective(s), target group(s) and approach of the mental health tools. For the assessment tool, we undertook a modified Delphi consensus process and used existing validated screening instruments. Both tools were compiled during two 2-day meeting. We also explored the appropriateness and preliminary reliability and validity of the assessment tool.

Sport Mental Health Assessment Tool 1 and Sport Mental Health Recognition Tool 1 The International Olympic Committee Sport Mental Health Assessment Tool 1 (SMHAT-1) was developed for sports medicine physicians and other licensed/registered health professionals to assess elite athletes (defined as professional, Olympic, Paralympic or collegiate level; aged 16 years and older) potentially at risk for or already experiencing mental health symptoms and disorders. The SMHAT-1 consists of: (i) triage with an athlete-specific screening tool, (ii) six subsequent disorder-specific screening tools and (iii) a clinical assessment (and related management) by a sports medicine physician or licensed/ registered mental health professional (eg, psychiatrist and psychologist). The International Olympic Committee Sport Mental Health Recognition Tool 1 (SMHRT-1) was developed for athletes and their entourage (eg, friends, fellow athletes, family and coaches).

Conclusion The SMHAT-1 and SMHRT-1 enable that mental health symptoms and disorders in elite athletes are recognised earlier than they otherwise would. These tools should facilitate the timely referral of those athletes in need for appropriate support and treatment.

\section{INTRODUCTION}

Mental health symptoms and disorders include those involving anxiety, depression or substance misuse. ${ }^{1}$ Their prevalence in elite athletes is substantial, and similar to their prevalence in the general population.
It ranges from $19 \%$ for alcohol misuse to $34 \%$ for anxiety/depression for active elite athletes and from $16 \%$ for distress to $26 \%$ for anxiety/depression for former elite athletes. ${ }^{1}$ During an elite sport career, generic and sport-specific stressors might increase the risk of mental health symptoms and disorders. ${ }^{2-4}$ Adverse life events, severe musculoskeletal injuries and related surgeries with long recovery periods are associated with mental health symptoms and disorders. ${ }^{2-4}$ Transitioning out of elite sport is also difficult for many former athletes. ${ }^{2-4}$

In 2017, the International Olympic Committee (IOC) selected an international expert panel comprising 27 individuals to thoroughly review the available scientific literature regarding mental health symptoms and disorders among active and former elite athletes (professional, Olympic or collegiate level). These 27 experts were invited by the IOC to a 2.5-day consensus meeting in November 2018 in Lausanne, Switzerland. Scientific and clinical findings were presented and discussed, ultimately leading to the International Olympic Committee consensus statement on mental health in elite athletes. ${ }^{5}$ One of the main recommendations embedded in the IOC consensus statement was the need to appropriately screen for mental health symptoms and disorders in elite athletes throughout their career to ensure that affected athletes ultimately receive the support and treatment they need. ${ }^{5}$ Following the consensus meeting, the IOC established the Mental Health Working Group (MHWG) with 11 international experts (10 of whom were involved in the IOC consensus statement) aiming in part to develop an assessment and recognition tool for the early identification of elite athletes potentially at risk for experiencing mental health symptoms and disorders. This article describes the evidence-based and practice-based development of the tools. It also presents the preliminary reliability and validity, utility and feasibility of the assessment tool.

\section{METHODS}

We applied the Intervention Mapping and Knowledge Transfer Scheme to develop the assessment and recognition tool. ${ }^{6-10}$ We undertook the following steps: (A) reviewing the scientific literature on mental health symptoms and disorders 
in elite athletes; (B) assessing the views of active and former elite athletes (inclusive of members of the athletes' entourage) regarding mental health symptoms and disorders; (C) formulating the objective(s), target group(s) and approach of the tools; (D) selecting the content of the tools and their development; and (E) assessing the appropriateness and preliminary reliability and validity of the assessment tool.

\section{Step A: Review of the scientific literature}

Authors of the IOC consensus statement conducted narrative and systematic reviews regarding the prevalence, diagnosis, screening, treatment and management of mental health symptoms and disorders in elite sports. Search strategies were applied in relevant electronic databases (eg, PubMed, SportDiscus, PSycINFO, Scopus and Cochrane) for articles published in November 2018, utilising predefined eligibility criteria. Data from the included articles were extracted within standardised extraction forms and risk of bias of the included studies was described. When applicable, meta-analyses were performed, and pooled estimates were calculated.

\section{Step B: Views of active and former elite athletes (including} members of the athletes' entourage)

We gathered the views of active and former elite athletes, inclusive of their entourage, on concepts related to mental health symptoms and disorders in elite athletes via a short electronic questionnaire (Mentimeter, Stockholm, Sweden) at (i) the IOC International Athletes' Forum (April 2019, Lausanne, Switzerland) and (ii) the International Paralympic Committee's Athletes' Forum (November 2019, Colorado Springs, USA). The attendees $(\mathrm{N}=360)$ were either (i) active and former elite athletes (as members of National Olympic Committee and National Paralympic Committee athletes' commissions), (ii) coaches in elite sports or (iii) representatives of sport governing bodies. The IOC MHWG submitted six questions and statements in English (sole language of these forums) to the audience as follows:

1. What is the first word that comes to your mind when you hear the words 'mental health symptoms'?

2. Which is the first group you turn to when you feel low?

3. Who is most important to the protection of athletes' mental health?

4. In elite sports, no one wants to talk about mental health problems (scored on 5-point scale, from 'strongly disagree' to 'strongly agree').

5. There is no recognition of mental health problems in my sport (scored on 5-point scale, from 'strongly disagree' to 'Strongly agree').

6. There should be routine screenings for mental health problems by medical staff in my sport ('yes' or 'no').

The IOC Medical and Scientific Committee provided institutional approval. Descriptive analyses (frequency) were conducted with the answers to these questions and statements.

\section{Step C: Objective(s), target group(s) and approach}

During a 2-day meeting of the IOC MHWG in April 2019 in Lausanne (Switzerland), we defined the objective(s), target group(s) and approach of the tools. Participants of the meeting were international experts with specialised training, skills and experience in a wide range of relevant domains, including but not limited to psychiatry, sports medicine, psychometrics and elite sport itself. We combined the information gathered from the scientific literature (step A) with the view of elite athletes and their entourage (step B), and relied on existing mental health screening and awareness tools used in sports for other domains such as concussion, harassment and abuse and relative energy deficiency in sport. ${ }^{11-13}$

\section{Step D: Content and development}

We selected the content and design of the tools during two 2-day meeting of the IOC MHWG held in Lausanne (Switzerland) in April and June 2019 (see step C). First, we considered the reproducibility, validity, practicality (ie, assessment time), use and applicability of existing screening instruments in the context of elite sports. Next, 15 independent experts gauged the appropriateness of the selected existing screening instruments through an expert rating process (ethical approval provided by the University of Washington's Institutional Review Board). These experts were either (1) authors of the International Olympic Committee consensus statement on mental health in elite atbletes and related specific narrative and systematic reviews of the scientific literature (see step A) or (2) licensed mental health professionals who are members of the Big Sky Sport Psychology Group and/or the Collegiate Clinical/Counselling Sport Psychology Association. Via an anonymous online survey, these experts were asked to indicate the extent to which they disagreed or agreed with the selection of the screening instruments on a 5-point Likert scale (from ' 1 ' as 'strongly disagree' to ' 5 ' as 'strongly agree').

Next, we conducted a modified Delphi consensus process to evaluate a provisional version of the assessment tool (ethical approval provided by the University of Washington's Institutional Review Board). ${ }^{14-19}$ Via an anonymous online survey, the experts were asked to provide quantitative and qualitative feedback on the seven components in this provisional version (introductory text and the six components labelled as 'Action'). The online survey contained an embedded screenshot of the specific tool component on which feedback was being elicited, followed by two questions: (i) how clinically useful is the specific component and (ii) how feasible is the specific component for its application in elite sport settings. Each question was scored on a 9-point scale (from ' 1 ' for 'not useful/feasible' to ' 9 ' for 'extremely useful/feasible') with the opportunity for open-ended written feedback. ${ }^{20}$ Descriptive statistics for both questions were calculated for each specific tool component, and open-ended feedback was compiled. A priori thresholds for component-level consensus were set as (1) mean score of greater than 7, (2) at least $70 \%$ of respondents with a score of greater than 7 and (3) a coefficient of variation (CV) of less than $0.50 .{ }^{20-22}$ The IOC MHWG and Delphi process facilitator (EK) reviewed and synthetised the results of the first round of Delphi voting, including reviewing open-ended participant comments to identify emergent themes related to lack of participant-rated utility or feasibility. ${ }^{23}$

Tool components meeting a priori thresholds for both usefulness and feasibility were not modified. Tool components not reaching numeric consensus thresholds were discussed, with a focus on themes emergent from the qualitative participant feedback. ${ }^{24}$ Following modifications, these steps were repeated.

\section{Step E: Appropriateness and preliminary reliability and validity}

We explored (i) the appropriateness of the assessment tool among sport medicine physicians and (ii) its preliminary reliability and validity among professional footballers.

Sport medicine physicians $(\mathrm{N}=43)$ participating in the International Federations Medical Commission Chairpersons Meeting (March 2020, Monaco; institutional approval provided by the IOC Medical and Scientific Committee) were asked to assess 
the appropriateness of the assessment tool following application to two hypothetical cases. Their evaluation was collected electronically (Mentimeter, Stockholm, Sweden) through eight statements (eg, 'The tool will be useful for health professionals working in the context of elite sports') rated on a 4-point scale (strongly disagree, disagree, agree and strongly agree). Participants also provided qualitative feedback .

The preliminary reliability and validity of the screening instruments selected for the assessment tool were evaluated through an observational study based on a cross-sectional design conducted among male-identified and female-identified professional footballers from Australian A-League and W-League (ethical approval provided by the Medical Ethics Review Committee of the Amsterdam University Medical Centers, location AMC; W18 340\#18.387). Participants (men: $N=149$, mean age: 24 years, mean career duration: 6 years; women: $N=132$, mean age: 23 years, mean career duration: 5 years) were asked to complete the screening instruments for the tool, in addition to the validated Kessler Distress questionnaire (K10). ${ }^{25}$ Internal consistency (degree of inter-relatedness among items) of each screening instrument was ascertained, being expressed with Cronbach's alpha ( $\alpha \geq 0.70$ considered as good; $0.60<\alpha<0.70$ considered as moderate; $r \leq 0.59$ considered as low and thresholds lower with fewer items). ${ }^{26}$ When applicable, convergent validity with K10 was calculated and expressed with Pearson correlation coefficients ( $r \geq 0.60$ considered as good; $0.60<r<0.30$ considered as moderate and $r \leq 0.30$ considered as low) while a series of area under receiver operating characteristic curves was undertaken to evaluate the existing cut-off scores relative to the K10 cutoff scores for moderate distress $(\mathrm{K} 10 \geq 16)$, high distress (K10 $\geq 22)$ and very high distress $(\mathrm{K} 10 \geq 30)$ separately (AUC $\geq 0.90$ considered as very good; AUC $\geq 0.80$ considered as good and AUC $\geq 0.70$ considered as fair). ${ }^{2627}$ Analysis of misclassification (false negative rate) between selected screening instruments was also undertaken.
Patient and public involvement

Active and former elite athletes, inclusive of their entourage (eg, athletes' representatives, coaches, representatives of governing bodies and sports medicine physicians), were involved in developing the tool and assessing its validation and appropriateness.

\section{RESULTS}

Step A: Reviewing the scientific literature

In total, 14689 citations were identified and screened by the international experts, leading ultimately to the International Olympic Committee consensus statement on mental health in elite athletes and 11 related publications. ${ }^{15}$ 28-37 All available evidence was published in two issues of the British Journal of Sports Medicine.

Step B: View of active and former elite athletes (including entourage)

The participants in the IOC International Athletes' Forum associated a wide range of words with the term 'mental health symptoms' (figure 1). Nearly $55 \%$ of respondents mentioned the word 'depression', 33\% the word 'stress', and 20\% the words 'anxiety' or 'pressure'. They reported that they would approach friends and fellow athletes (50\%), family (40\%) and coaches $(8 \%)$ when experiencing mental health symptoms. Participants identified coaches (55\%), parents and friends (25\%) and medical team $(9 \%)$ as important facilitators of athletes' mental health. Nearly two-thirds of the participants agreed that talking about mental health symptoms was taboo in elite sports and that there was no recognition of these symptoms in their sport. Ninety per cent were in favour of routine screenings by the medical team for mental health symptoms in their sport.

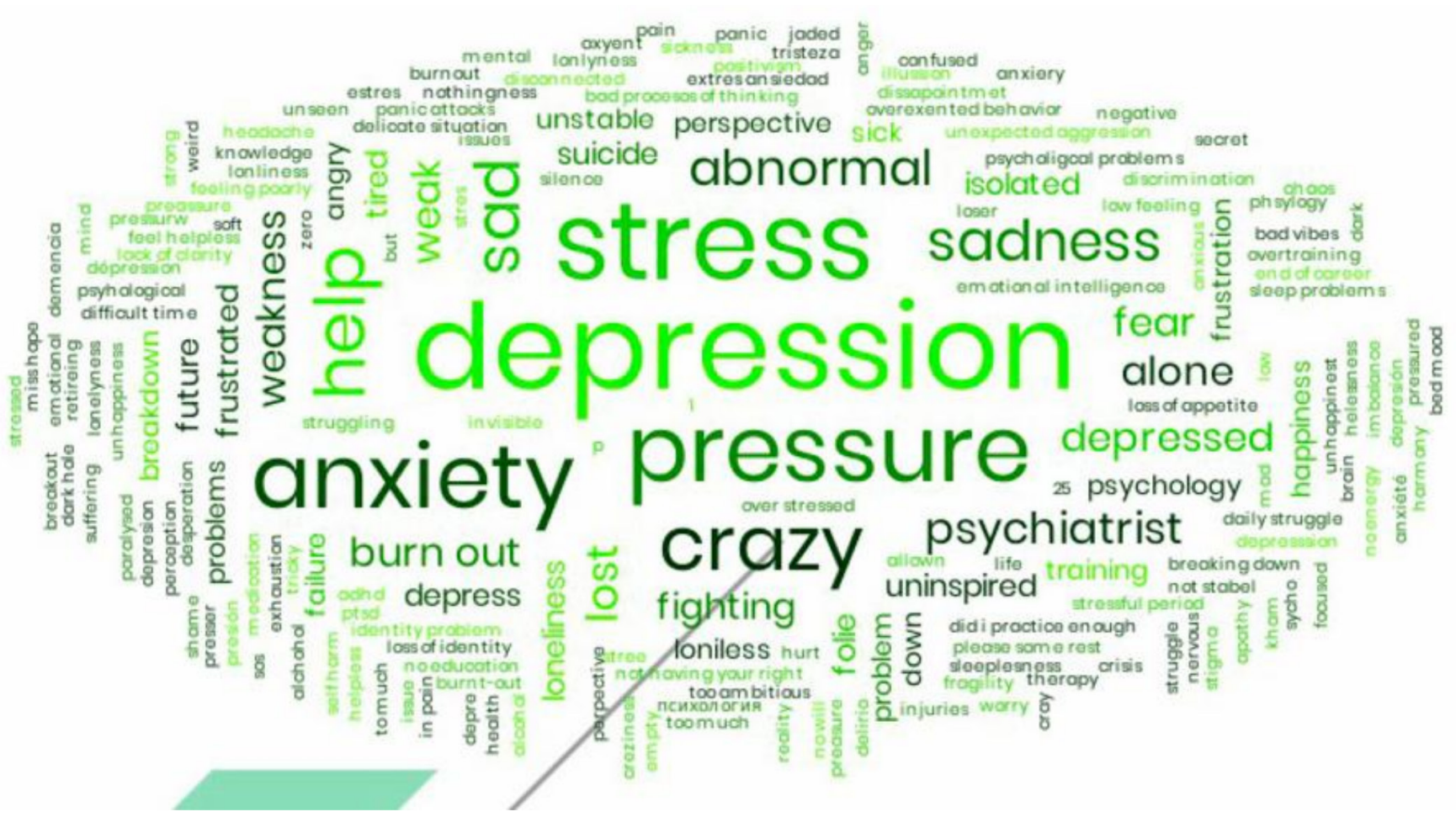

Figure 1 Word cloud of the view of athletes (including entourage) about mental health symptoms. 
Step C: Objective(s), target group(s) and approach

Based on steps A and B, the IOC MHWG formulated the following objective for the assessment tool: to assess elite athletes (defined as professional, Olympic, Paralympic or collegiate level; aged 16 years and older) potentially at risk for (ie, exposed to one or more stressors), or already experiencing, mental health symptoms and disorders to facilitate timely management and/ or referral to adequate support and/or treatment. We established that the assessment tool should be used only by sports medicine physicians and other licensed/registered health professionals, and we defined a three-step approach (step 1: triage; step 2: screening and step 3: intervention and (re)assessment).

\section{Step D: Content and development}

We selected an existing validated screening instrument (available freely in the public domain) for the triage step (step 1) and six existing validated screening instruments (available freely in the public domain), related to the most prevalent mental health symptoms in elite sports, for the screening step (step 2). ${ }^{15}$ Combined with a final section on intervention and $r(e)$ assessment (step 3), we developed a provisional version of the assessment tool called the IOC Sport Mental Health Assessment Tool (SMHAT).

Each of the screening tools included in the SMHAT were rated as 'appropriate' or 'very appropriate' (scores of 4 or 5 on a 5 -point scale) by more than $73 \%$ of experts, and five of the seven had mean scores of $>4.00$. The two lowest scoring scales were athlete distress, as measured by the Athlete Psychological Strain Questionnaire (APSQ: mean $=3.80, \mathrm{SD}=1.15,73 \%$ 'appropriate' or 'very appropriate'), and depression, as measured by the Patient Health Questionnaire-9 (PHQ-9) (mean=3.93, $\mathrm{SD}=1.33,80 \%$ 'appropriate' or 'very appropriate'). Despite the expressed concerns about the APSQ's sensitivity and specificity in identifying athletes who require further screening, the IOC MHWG elected to retain this measure because evidence about the APSQ's psychometrics was imminently to be published. ${ }^{37} 38$ Concern was also expressed about the utility of the PHQ-9 to measure more subtle subclinical issues. The IOC MHWG elected to retain the PHQ-9 because it is one of the commonly used instruments in the context of elite sports which has been validated across different cultures and languages.

In the first round of the Delphi consensus process, one component that did not meet a priori thresholds for both utility and feasibility was 'step 3b' (clinical assessment and management, mean utility score $=6.87, \mathrm{SD}=2.23,67 \%>7, \mathrm{CV}=32.50$; mean feasibility score $=6.93, \mathrm{SD}=1.28,73 \%>7, \mathrm{CV}=18.46$ ). There were concerns about the required expertise for this step. No alteration to this component was made because the IOC MHWG strongly believed that sport medicine physicians working with elite athletes should have sufficient competency to conduct a clinical assessment and define a management plan. Two other components did not meet thresholds for consensus feasibility. The introduction $($ mean $=6.60, \mathrm{SD}=1.40,53 \%>7, \mathrm{CV}=21.27$ ) was described as being too long and difficult to read in the context of clinical practice. In response, the IOC MHWG edited this section to streamline and facilitate understanding. Concerns were also expressed about the additional screening tools for mental health symptoms and disorders (mean $=6.60, \mathrm{SD}=2.03$, $67 \%>7, \mathrm{CV}=30.73$ ). The IOC MHWG elected to not change this section because these additional screening tools are not mandatory and are only suggestions for clinicians to consider.

The revised SMHAT was shared with the same pool of experts for an additional round of rating on the two edited components.
Utility and feasibility of the edited introduction exceeded consensus thresholds. Utility of step $3 \mathrm{~b}$ (clinical assessment and management) also exceeded consensus thresholds (mean $=8.14$, $\mathrm{SD}=1.21,85.71 \%>7, \mathrm{CV}=14.92$ ), while feasibility did not exceed the threshold (mean $=6.71, \mathrm{SD}=2.06,71.43 \%>7$, $\mathrm{CV}=30.66$ ). Feasibility concerns for this section continued to relate to the level of training of the person making this assessment. The IOC MHWG elected to leave this component unaltered, in recognition of the challenge of having access to licensed mental health professionals in sport settings. ${ }^{5}$ Another consideration is that we developed a separate tool to facilitate recognition and triage of mental health concerns for use by non-clinical personnel.

\section{The Sport Mental Health Assessment Tool 1}

Based on three steps (figure 2), the SMHAT-1 (first version; intended to be updated in the future) allows for early stage identification of those athletes in need of mental health treatment. The SMHAT-1 can be used by sports medicine physicians and other licensed/registered health professionals, but the clinical assessment (and related management) within the SMHAT-1 (see step 3 b) must be conducted by sport medicine physicians and/ or licensed/registered mental health professionals, including clinically trained sport psychologists. Physical therapists, athletic trainers and not clinically trained sport psychologists working with a sports medicine physician can use the SMHAT-1, but any guidance or intervention should remain the responsibility of their sports medicine physician.

\section{Step 1: triage}

The APSQ assesses sport-related psychological distress and is used for a first triage. ${ }^{38}$ The APSQ is a brief, self-report rating scale specific to the sport context and relying on 10 items (eg, 'During the past 4 weeks, I could not stop worrying about injury or my performance') scored on a 5-point scale (from 'none of the time' (1) to 'all of the time' (5)). ${ }^{37}$ A total score ranging from 10 to 50 is calculated by summing the answers on the 10 items, with a score of 17 or more indicating an elevated or high risk for psychological distress. ${ }^{38} 39$ The APSQ has been validated in male and female athletes (area under curve value >0.90). ${ }^{38} 39$ In case of a negative triage, no further action is needed, while a positive triage leads to the subsequent step (step 2: Screening).

\section{Step 2: screening}

In case of a positive triage, athletes proceed to step 2 and are assessed on six disorder-specific screening questionnaires:

- General Anxiety Disorder-7 (GAD-7): assesses the presence of symptoms of anxiety. ${ }^{40}$

- Patient Health Questionnaire-9 (PHQ-9): assesses the presence of symptoms of depression. ${ }^{41} 42$

- Athlete Sleep Screening Questionnaire (ASSQ): assesses the presence of sleep disturbance. ${ }^{43-45}$

- Alcohol Use Disorders Identification Test Consumption (AUDIT-C): assesses the presence of alcohol misuse. ${ }^{46-48}$

- Cutting Down, Annoyance by Criticism, Guilty Feeling, and Eye-openers Adapted to Include Drugs (CAGE-AID): assesses the presence of substance misuse, being slightly adapted for the SMHAT (no focus on alcohol use as already explored with the AUDIT-C; additional question to explore which substance was used). ${ }^{49-54}$

- Brief Eating Disorder in Athletes Questionnaire (BEDA-Q): assesses the presence of disordered eating. ${ }^{55}$ 


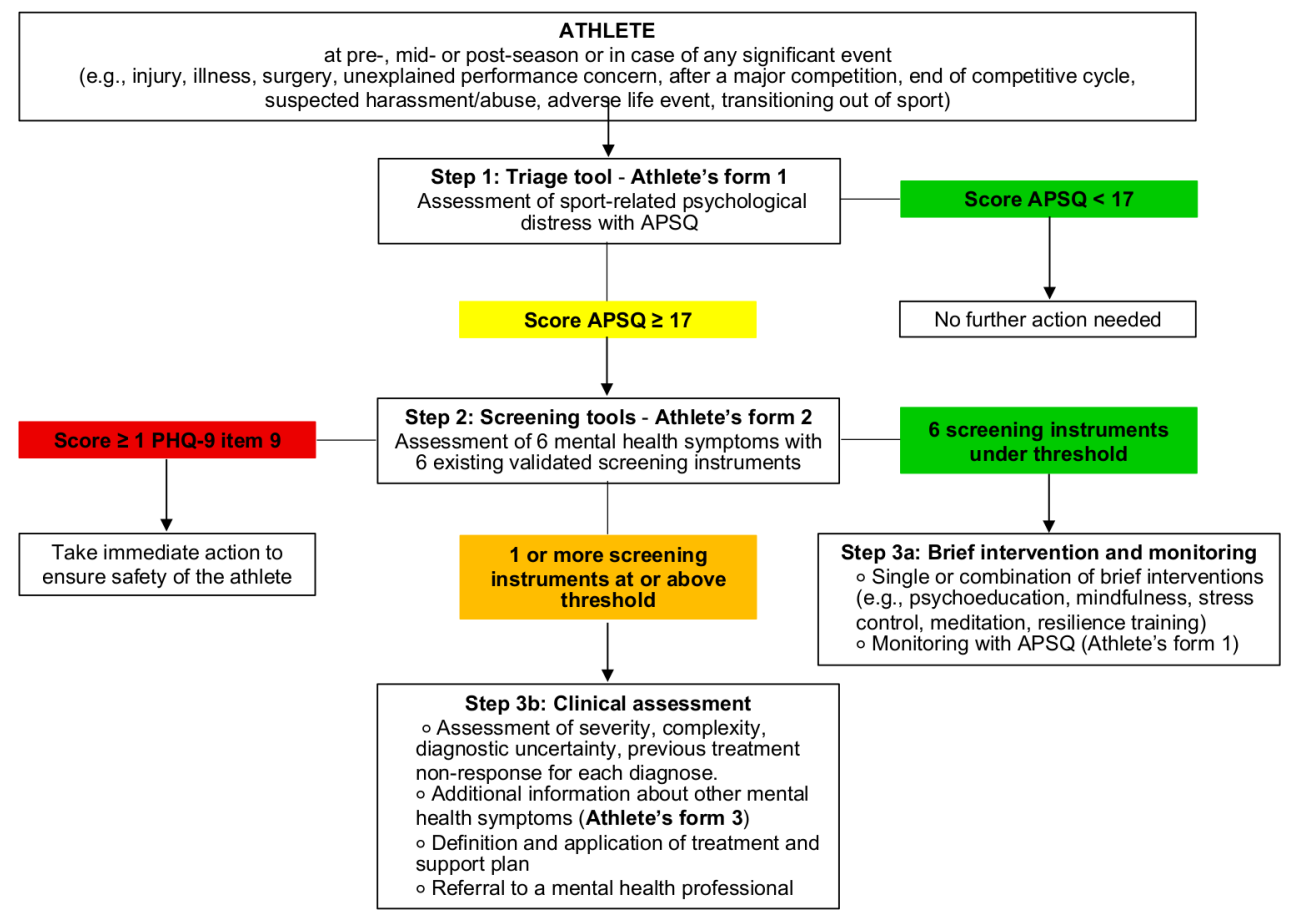

Figure 2 Flow chart of the International Olympic Committee Sport Mental Health Assessment Tool 1.

Further information about these existing validated screening questionnaires (including psychometric properties) is presented in online supplementary material. If all screening questionnaires are negative, the administrator proceeds to step 3 a (brief intervention and monitoring, given the report of elevated psychological distress at step 1: triage). If one or more screening questionnaires are positive (or if a positive answer is given on item 9 of the PHQ-9), the administrator proceeds to step $3 \mathrm{~b}$ (clinical assessment and management).

\section{Step 3a: brief intervention and monitoring}

Based on the athlete's history/record and on the information provided in steps 1 and 2, the administrator might refer the athlete to a brief intervention such as psychoeducation, mindfulness, meditation, mental skills training or stress control. Depending on the progress of the athlete and after the completion of the brief intervention(s), the administrator should consider readministering the APSQ. In the case of a negative APSQ, no further action is needed, while another positive APSQ leads to step $3 \mathrm{~b}$ (assessment and management).

\section{Step 3b: clinical assessment and management}

Considering the previous outcomes (steps 1 and 2), a comprehensive clinical assessment is conducted by a sport medicine physician and/or licensed/registered mental health professional (eg, psychiatrist and clinical psychologist) to obtain additional relevant information (eg, mental health history, history of and/ or presence of harassment/abuse within or outside of sports) and ultimately to identify a clinical diagnosis. For each of these diagnoses, severity and complexity, as well as diagnostic uncertainty and previous treatment non-response, are assessed. If the sports medicine physician does not feel comfortable determining the severity and/or complexity of a given athlete's diagnosis, then referral to a licensed/registered mental health professional may be made. Based on all available information, the sport medicine physician and/or licensed/registered mental health professional chooses one of the following three actions:
1. In cases that are neither severe nor complex and where there is no diagnostic uncertainty nor history of previous non-responsive to treatment, then treatment/support can be provided by a sports medicine/primary care physician. The physician should refer to the International Olympic Committee consensus statement on mental health in elite athletes for specific guidance on treatment modalities. ${ }^{5}$

2. In cases of diagnostic uncertainty or when further information might be useful, additional screening questionnaires for other mental health symptoms and disorders, including but not limited to attention-deficit/hyperactivity disorder, bipolar disorder, post-traumatic stress disorder, gambling disorder and/or psychosis, can be considered prior to definitive diagnosis and the creation of a management/intervention plan. ${ }^{56-66}$

3. In cases that are severe, complex, diagnostically uncertain even after additional screening is completed and/or nonresponsive to treatment, we recommend referral of the athlete to a licensed/registered mental health professional (eg, clinical psychologist or psychiatrist).

The SMHAT-1 should be embedded within the precompetition period (ie, ideally a few weeks after the start of sport training), as well as within the mid-season and end-season period. The SMHAT-1 can also be used when the athlete experiences any significant life event such as major injury/illness, surgery, unexplained performance concern, suspected harassment/abuse, after a major competition, at the end of a competitive cycle, or when transitioning out of sport. The SMHAT-1 is available as a paper version (online supplementary appendix); however, the triage and screening (steps 1 and 2) are designed to be ideally embedded in a privacy-secured online platform. The SMHAT-1 can be freely copied in its current form for distribution to individuals, teams, groups and organisations. Any revision requires the approval by the IOC MHWG. To strengthen its multicultural use, we encourage the translation of the SMHAT-1 in collaboration with the IOC MHWG. The SMHAT-1 should not be re-branded or sold for commercial gain. 
The Sport Mental Health Recognition Tool 1

Since the athletes' entourage (eg, friends, fellow athletes, family and coaches) were identified as essential supports for athletes mental health (see step 3b), the IOC MHWG developed the IOC Sport Mental Health Recognition Tool 1 (SMHRT-1, first version; intended to be updated in the future), a recognition tool to be used by athletes coaches, family members and all other members of the athlete's entourage. The objective of the SMHRT-1 is to facilitate early detection of mental health symptoms in elite athletes (professional, Olympic, Paralympic or collegiate level; aged 16 years and older) to promote helpseeking for those athletes in need of assistance from a sports medicine physician or other licensed/registered health professional and to facilitate further assessment and subsequent treatment as applicable.

The SMHRT-1 relies on the observation of significant and/or persistent thoughts, feelings, behaviours and/or physical changes in athletes. In cases where those are observed, the athletes should be directed to a sports medicine physician or licensed/registered health professional for mental health screening with the SMHAT-1. Within the SMHRT-1, several items constituting red flags were also identified (eg, comments related to harming self or others), which warrant immediate help-seeking and possible crisis management. The SMHRT-1 is available in paper format (online supplementary appendix) and can be freely copied in its current form for distribution to individuals, teams, groups and organisations. Any revision requires the specific approval by the IOC MHWG while any translation should be reported to the IOC MHWG. The SMHRT-1 should not be rebranded or sold for commercial gain.

\section{Step E: Appropriateness and preliminary reliability and validity}

More than $90 \%$ of participants in the International Federations Medical Commission Chairpersons Meeting agreed that the SMHAT-1 would be useful for sports medicine physicians and other licensed/registered health professionals working in the context of elite sports. Around 55\% of respondents were positive about the ease of use of the SMHAT-1, mentioning especially that translation in different languages is needed. Nearly 90\% stated that the three-step approach of the SMHAT-1 was appropriate, while each single step was also assessed positively: 93\% for step 1 (triage); 100\% for step 2 (screening); $85 \%$ for step 3a (brief intervention); $83 \%$ for step $3 \mathrm{~b}$ (clinical assessment); $63 \%$ for additional screening in step $3 \mathrm{~b}$.

Internal consistency was moderate to good for the APSQ (men: $\alpha=0.81$; women: $\alpha=0.84$ ), GAD-7 (men: $\alpha=0.91$; women: $\alpha=0.88$ ), PHQ-9 (men: $\alpha=0.87$; women: $\alpha=0.83$ ), ASSQ (men: $\alpha=0.72$; women: $\alpha=0.67$ ), AUDIT-C (men: $\alpha=0.74$; women: $\alpha=0.66$ ) and CAGE-AID (men: $\alpha=0.69$; women: $\alpha=0.66$ ). Internal consistency was low for the BEDA-Q (men: $\alpha=0.59$; women: $\alpha=0.66$ ). Convergent validity between APSQ and K10 was good to very good in both male-identified and female-identified professional footballers $(r=0.70-0.73$, $\mathrm{p}<0.01 ; \mathrm{AUC}=0.87-0.96, \mathrm{p}<0.01)$. The APSQ applied at step 1 (triage) identified $57.1 \%(\mathrm{~N}=160)$ of the sample requiring progression to step 2 (screening). Overall, the APSQ performed well in case detection for the six screening instruments at step 2 (table 1). The APSQ correctly detected all positive screened cases for the GAD-7, PHQ-9 and CAGE-AID, while only a small proportion of cases was misclassified for the AASQ $(5 \%)$, AUDIT-C (16\%) and BEDA-Q (11\%).
Table 1 Misclassification of the APSQ at step 1 (triage) for six instruments at step 2 (screening)

\begin{tabular}{lll}
\hline & $\begin{array}{l}\text { Positive cases detected at } \\
\text { step 1 (APSQ) and at step 2 }\end{array}$ & $\begin{array}{l}\text { Positive cases not detected at step } \\
\text { (APSQ) but detected at step 2 }\end{array}$ \\
\hline GAD-7 & $\mathrm{N}=18(6 \%)$ & $\mathrm{N}=0(0 \%)$ \\
PHQ-9 & $\mathrm{N}=24(9 \%)$ & $\mathrm{N}=0(0 \%)$ \\
ASSQ & $\mathrm{N}=61(22 \%)$ & $\mathrm{N}=14(5 \%)$ \\
AUDIT-C & $\mathrm{N}=88(32 \%)$ & $\mathrm{N}=44(16 \%)$ \\
CAGE-AID & $\mathrm{N}=5(2 \%)$ & $\mathrm{N}=0(0 \%)$ \\
BEDA-Q & $\mathrm{N}=77(28 \%)$ & $\mathrm{N}=32(11 \%)$
\end{tabular}

APSO, Athlete Psychological Strain Questionnaire; ASSQ, Athlete Sleep Screening Questionnaire; AUDIT-C, Alcohol Use Disorders Identification Test Consumption; BEDA-Q, Brief Eating Disorder in Athletes Questionnaire; CAGE-AID, Cutting Down, Annoyance by Criticism, Guilty Feeling, and Eye-openers Adapted to Include Drugs; GAD-7, General Anxiety Disorder-7; N, number; PHQ-9, Patient Health Questionnaire-9.

\section{DISCUSSION}

In response to a recommendation of the International Olympic Committee consensus statement on mental health in elite athletes, the SMHAT-1 and SMHRT-1 were developed to provide tools to assess and manage mental health symptoms and disorders in elite athletes throughout their sporting career. For the SMHAT-1, the IOC MHWG strived to select existing validated screening questionnaires for mental health symptoms and disorders that were developed for and validated in an (elite) athlete population. However, such questionnaires are rather scarce. Within the SMHAT-1, only 3 out of the 12 screening questionnaires are athlete-specific. One of them is the APSQ, which is used as triage to assess sport-related psychological distress. ${ }^{38} 39$ The APSQ was recently developed for and validated in professional athletes, focusing particularly on the sport environment. Therefore, it is possible that mental health symptoms and disorders that manifest outside the sport environment might not be captured with this questionnaire. Another screening questionnaire developed for and validated in an (elite) athlete population is the BEDA-Q, which assesses the presence of disordered eating. ${ }^{55}$ However, the BEDA-Q has no established cut-off, and therefore the authors proposed a cut-off of 4 or more based on the data presented in this article. It is important to emphasise that a positive screening on any disorder-specific screening questionnaires does not automatically provide a diagnosis for the disorder, but it is a prompt for further clinical evaluation. For the other screening questionnaires that were developed for and validated in non-athlete populations, their psychometric properties (especially sensitivity and specificity) might differ when used in an (elite) athlete population.

The SMHAT-1 and SMHRT-1 are the first versions of the tools to identify elite athletes at risk for experiencing mental health symptoms and disorders. Analogous to sport concussion and its assessment (SCAT) and recognition (CRT) tools, ${ }^{11}$ the IOC MHWG intends to revise the SMHAT-1 and SMHRT-1 in the future as needed, concurrent with an anticipated updating of the International Olympic Committee consensus statement on mental health in elite athletes. Until then, the IOC MHWG recommends further validation of the SMHAT-1's underlying screening questionnaires, and the analysis of practicality and utility in a variety of elite athlete populations across different sports and countries.

The SMHAT- 1 should be part of a regular screening programme, in parallel with, for example, musculoskeletal and cardiovascular screening. As the use of the SMHAT-1 is likely to increase the number of referrals, it is important to have sport-specific mental health supports in place for the care of athletes. We strongly recommend collaboration between sport medicine physicians and mental health professionals (eg, psychologists and psychiatrists). 
Furthermore, education about mental health symptoms and disorders in elite athletes as well as the use of the SMHAT-1 has been developed by the IOC to improve the clinical competency of sports medicine physicians and other licensed/registered health professionals. Ideally this education should be embedded within their respective professional school curricula. For all other members of the athletes' entourage, the SMHRT-1 can be used to increase focus on the mental health of elite athletes.

Several limitations of our approach should be acknowledged. First, information gathered through questionnaires in the SMHAT-1 remains self- reported and thus its external validity depends on the accuracy of the respondent's answers. Second, some of the existing validated screening questionnaires selected for the SMHAT- 1 were developed for and validated in non-athlete populations. Third, the triage step in the SMHAT- 1 relies on the APSQ. While this questionnaire includes athlete-specific questions within an athletic context and has been validated in several populations of elite athletes, the APSQ (as most other existing screening instruments) does not reach $100 \%$ sensitivity and $100 \%$ specificity. This implies the possibility of obtaining false negatives and false positives in the triage step of the SMHAT-1. Fourth, the SMHAT-1 should be used by sports medicine physicians and other licensed/ registered health professionals, while subsequent referral to a clinical psychologist or psychiatrist in particular cases is advised. In some countries, such a referral might not always be possible. Fifth, the SMHAT-1 and SMHRT-1 are currently available only in English. Translations (and related validation studies) should be made available in the future, which aligns with the ambition of the IOC. Lastly, the SMHAT-1 was preliminarily validated among professional footballers (men and women) from one country.

What are the findings?

- The International Olympic Committee Sport Mental Health Assessment Tool 1 (IOC SMHAT-1) was developed to identify elite athletes at risk for or already experiencing mental health symptoms and disorders, to facilitate timely referral of those in need to adequate support and treatment.

- IOC SMHAT-1 should be used by sports medicine physicians and other licensed/registered health professionals during the precompetition period (ie, ideally a few weeks after the start of sport training) and when any significant event for an athlete occurs (eg, major injury/illness, unexplained performance concern, end of competitive cycle, suspected harassment/abuse, adverse life event and transitioning out of sport).

- The IOC Sport Mental Health Recognition Tool 1 was developed for athletes, coaches, family members and all other members of the athlete's entourage to facilitate early detection of mental health symptoms in athletes to promote help-seeking for those in need.

\section{How might it impact on clinical practice in the future?}

- The use of the SMHAT-1 by licensed/registered health professionals (especially sports medicine physicians) and the SMHRT-1 by athletes and all members of their entourage should enable the identification of elite athletes at risk for experiencing mental health symptoms and disorders and facilitate the timely referral of those athletes in need for appropriate support and treatment.
Future validation studies should be conducted among athletes from other sport disciplines and cultures, while the use and application of the SMHAT-1 longitudinally should be evaluated. This is the intention of the IOC and its MHWG.

\section{CONCLUSION}

Following the International Olympic Committee consensus statement on mental health in elite athletes, we developed the SMHAT-1 and SMHRT-1 to identify elite athletes at risk for or already experiencing mental health symptoms and/or disorders. These tools should facilitate the timely referral of those athletes in need for appropriate support and treatment. This initiative aligns with the IOC's commitment to improve the mental health of elite athletes.

\section{Author affiliations}

${ }^{1}$ Amsterdam UMC, Univ of Amsterdam, Department of Orthopaedic Surgery, Amsterdam Movement Sciences, Amsterdam, The Netherlands

${ }^{2}$ Amsterdam Collaboration on Health \& Safety in Sports (ACHSS), Amsterdam UMC IOC Research Center of Excellence, Amsterdam, The Netherlands

${ }^{3}$ Athletes' Commission, International Olympic Committee, Lausanne, Switzerland ${ }^{4}$ Department of Physical Medicine and Rehabilitation, Spaulding Rehabilitation Hospital and Brigham and Women's Hospital, Harvard Medical School, Boston, Massachusetts, USA

${ }^{5}$ Sports Department, International Olympic Committee, Lausanne, Switzerland

${ }^{6}$ Regional Affective Disorders Service, Northumberland Tyne and Wear NHS Foundation Trust, Newcastle, UK

${ }^{7}$ Department of Sport and Exercise Sciences, The University of Sunderland, Sunderland, UK

${ }^{8}$ Orthopedic Clinic, University of Oslo, Oslo, Norway

${ }^{9}$ Medical and Scientific Department, International Olympic Committee, Lausanne, Switzerland

${ }^{10}$ National Collegiate Athletic Association (NCAA), Indianapolis, Indiana, USA ${ }^{11}$ Department of Pediatrics, University of Washington, Seattle, Washington, USA

${ }^{12}$ Center for Child Health, Behavior and Development, Seattle Children's Research Institute, Seattle, Washington, USA

${ }^{13}$ Department of Psychiatry, University of Maryland School of Medicine, Baltimore, Maryland, USA

${ }^{14}$ Department of Family Medicine - Sport, McMaster University, Hamilton, Ontario, Canada

${ }^{15}$ Orygen, The National Centre of Excellence in Youth Mental Health, Melbourne, Victoria, Australia

${ }^{16}$ Centre for Youth Mental Health, University of Melbourne, Melbourne, Victoria, Australia

${ }^{17}$ Athletic Medicine, University Health Services, Princeton University, Princeton, New Jersey, USA

${ }^{18}$ Department of Psychiatry, University of Wisconsin Madison School of Medicine and Public Health, Madison, Wisconsin, USA

Twitter Vincent Gouttebarge @vgouttebarge, Cheri Blauwet @CABlauwet, Lars Engebretsen @larsengebretsen, Margo Mountjoy @margo.mountjoy and Margot Putukian@Mputukian

Acknowledgements The authors thank the other participants in the International Olympic Committee consensus meeting on Mental Health in Elite Athletes, including Cindy Miller Aron, David Baron, Antonia Baum, Joao Mauricio Castaldelli-Maia, Jeff Derevensky, Ira Glick, Paul Gorczynski, Michael Grandner, Mary Hitchcock, Doug Hyun Han, Aslihan Polat, Allen Sills, Torbjorn Soligard, Todd Stull, Leslie Swartz and Li Jing Zhu, for their extensive review of the scientific literature. The authors are also thankful to the elite athletes and experts who participated in this study.

Contributors All authors contributed to the development of the Sport Mental Health Assessment Tool 1 and Sport Mental Health Recognition Tool 1. The Delphi consensus process was led by EK, while the appropriateness and preliminary validity of the tool was led by VG. VG was responsible for the preparation of the manuscript. All other authors were responsible for the critical review of the manuscript. All authors read and approved the final version of the manuscript.

Funding The authors have not declared a specific grant for this research from any funding agency in the public, commercial or not-for-profit sectors.

Competing interests None declared.

Patient and public involvement Patients and/or the public were involved in the design, or conduct, or reporting, or dissemination plans of this research. Refer to the Methods section for further details.

Patient consent for publication Not required. 
Ethics approval Ethical approval was provided by the University of Washington's Institutional Review Board and the Medical Ethics Review Committee of the Amsterdam University Medical Centers (location AMC), while institutional approval was provided by the IOC Medical and Scientific Committee.

Provenance and peer review Not commissioned; externally peer reviewed. Data availability statement No data are available.

Open access This is an open access article distributed in accordance with the Creative Commons Attribution Non Commercial (CC BY-NC 4.0) license, which permits others to distribute, remix, adapt, build upon this work non-commercially, and license their derivative works on different terms, provided the original work is properly cited, appropriate credit is given, any changes made indicated, and the use is non-commercial. See: http://creativecommons.org/licenses/by-nc/4.0/.

\section{ORCID iDs}

Vincent Gouttebarge http://orcid.org/0000-0002-0126-4177

Alan Currie http://orcid.org/0000-0001-5617-4868

Brian Hainline http://orcid.org/0000-0002-0233-2434

David McDuff http://orcid.org/0000-0003-4088-3920

Margo Mountjoy http://orcid.org/0000-0001-8604-2014

Margot Putukian http://orcid.org/0000-0002-1478-8068

\section{REFERENCES}

1 Gouttebarge V, Castaldelli-Maia JM, Gorczynski P, et al. Occurrence of mental health symptoms and disorders in current and former elite athletes: a systematic review and meta-analysis. Br J Sports Med 2019:53:700-6.

2 Arnold R, Fletcher D. A research synthesis and taxonomic classification of the organizational stressors encountered by sport performers. J Sport Exerc Psychol 2012;34:397-429

3 Engel GL. The need for a new medical model: a challenge for biomedicine. Science 1977;196:129-36

4 Wylleman P, Reints A. A lifespan perspective on the career of talented and elite athletes: perspectives on high-intensity sports. Scand J Med Sci Sports 2010;20 Suppl 2:88-94.

5 Reardon CL, Hainline B, Miller Aron C, et al. International Olympic Committee consensus statement on mental health in elite athletes. Br J Sports Med 2019:53:667-99.

6 Bartholomew LK, Parcel GS, Kok G. Intervention mapping: a process for developing theory- and evidence-based health education programs. Health Educ Behav 1998:25:545-63.

7 Verhagen E, Voogt N, Bruinsma A, et al. A knowledge transfer scheme to bridge the gap between science and practice: an integration of existing research frameworks into a tool for practice. Br J Sports Med 2014;48:698-701.

8 Gouttebarge V, Cowie C, Goedhart E, et al. Educational concussion module for professional footballers: from systematic development to feasibility and effect. $B M J$ Open Sport Exerc Med 2019;5:e00490.

9 Pas HIMFL, Bodde S, Kerkhoffs GMMJ, et al. Systematic development of a tennis injury prevention programme. BMJ Open Sport Exerc Med 2018:4:e000350

10 Gouttebarge V, Goedhart E, Kerkhoffs G. Empowering the health of retired professional footballers: the systematic development of an after career consultation and its feasibility. BMJ Open Sport Exerc Med 2018;4:e000466.

11 McCrory P, Meeuwisse W, Dvořák J, et al. Consensus statement on concussion in sport-the $5^{\text {th }}$ international conference on concussion in sport held in Berlin, October 2016. Br J Sports Med 2017; 51:838-47.

12 Mountjoy M, Brackenridge C, Arrington M, et al. International Olympic Committee consensus statement: harassment and abuse (non-accidental violence) in sport. $\mathrm{Br}$ J Sports Med 2016;50:1019-29.

13 Mountjoy M, Sundgot-Borgen J, Burke L, et al. RED-S cat. relative energy deficiency in sport (RED-S) clinical assessment tool (cat). Br I Sports Med 2015;49:421-3.

14 Adler M, Ziglio E. Gazing into the oracle: the Delphi method and its application to social policy and public health. London: Jessica Kingsley Publishers, 1996.

15 McKenna HP. The Delphi technique: a worthwhile research approach for nursing? J Adv Nurs 1994;19:1221-5

16 Powell C. The Delphi technique: myths and realities. J Adv Nurs 2003;41:376-82

17 Chang AM, Gardner GE, Duffield C, et al. A Delphi study to validate an advanced practice nursing tool. J Adv Nurs 2010;66:2320-30.

18 Humphrey-Murto S, Varpio L, Wood TJ, et al. The use of the Delphi and other consensus group methods in medical education research: a review. Acad Med 2017;92:1491-8.

19 Jorm AF. Using the Delphi expert consensus method in mental health research. Aust $N$ Z J Psychiatry 2015;49:887-97.

20 Rivara FP, Ennis SK, Mangione-Smith R, et al. Quality of care indicators for the rehabilitation of children with traumatic brain injury. Arch Phys Med Rehabil 2012;93:381-5
21 Li Y, Ehiri J, Hu D, et al. Framework of behavioral indicators for outcome evaluation of TB health promotion: a Delphi study of TB suspects and TB patients. BMC Infect Dis 2014;14:268.

22 Shah HA, Kalaian SA. Which is the best parametric statistical method for analyzing Delphi data? J Mod App Stat Meth 2009;8:226-32.

23 Holey EA, Feeley JL, Dixon J, et al. An exploration of the use of simple statistics to measure consensus and stability in Delphi studies. BMC Med Res Methodol 2007;7:52.

24 Joseph-Williams N, Newcombe R, Politi M, et al. Toward minimum standards for certifying patient decision AIDS: a modified Delphi consensus process. Med Decis Making 2014;34:699-710.

25 Kessler RC, Andrews G, Colpe LJ, et al. Short screening scales to monitor population prevalences and trends in non-specific psychological distress. Psychol Med 2002;32:959-76

26 Prinsen CAC, Mokkink LB, Bouter LM, et al. COSMIN guideline for systematic reviews of patient-reported outcome measures. Qual Life Res 2018;27:1147-57.

27 Ekelund S. Roc Curves-What are they and how are they used? Point Care 2012;11:16-21.

28 Aron CM, Harvey S, Hainline B, et al. Post-Traumatic stress disorder (PTSD) and other trauma-related mental disorders in elite athletes: a narrative review. Br I Sports Med 2019;53:779-84

29 Castaldelli-Maia JM, Gallinaro JGdeME, Falcão RS, et al. Mental health symptoms and disorders in elite athletes: a systematic review on cultural influencers and barriers to athletes seeking treatment. Br I Sports Med 2019;53:707-21.

30 Currie A, Gorczynski P, Rice SM, et al. Bipolar and psychotic disorders in elite athletes: a narrative review. Br J Sports Med 2019:53:746-53.

31 Currie A, McDuff D, Johnston A, et al. Management of mental health emergencies in elite athletes: a narrative review. Br I Sports Med 2019:53:772-8.

32 Han DH, McDuff D, Thompson D, et al. Attention-Deficit/Hyperactivity disorder in elite athletes: a narrative review. Br J Sports Med 2019:53:741-5.

33 Kroshus E, Wagner J, Wyrick D, et al. Wake up call for collegiate athlete sleep: narrative review and consensus recommendations from the NCAA Interassociation Task force on sleep and wellness. Br J Sports Med 2019;53:731-6.

34 McDuff D, Stull T, Castaldelli-Maia JM, et al. Recreational and ergogenic substance use and substance use disorders in elite athletes: a narrative review. Br J Sports Med 2019;53:754-60

35 Rice SM, Gwyther K, Santesteban-Echarri O, et al. Determinants of anxiety in elite athletes: a systematic review and meta-analysis. Br J Sports Med 2019;53:722-30.

36 Stillman MA, Glick ID, McDuff D, et al. Psychotherapy for mental health symptoms and disorders in elite athletes: a narrative review. Br I Sports Med 2019:53:767-71.

37 Swartz L, Hunt X, Bantjes J, et al. Mental health symptoms and disorders in Paralympic athletes: a narrative review. Br J Sports Med 2019:53:737-40.

38 Rice SM, Parker AG, Mawren D, et al. Preliminary psychometric validation of a brief screening tool for athlete mental health among male elite athletes: the athlete psychological strain questionnaire. Int I Sport Exerc Psychol 2019:6:1-16.

39 Rice S, Olive L, Gouttebarge V, Clifton P, et al. Mental health screening: severity and cut-off point sensitivity of the athlete psychological strain questionnaire in male and female elite athletes. BMJ Open Sport Exerc Med 2020;6:e000712.

40 Spitzer RL, Kroenke K, Williams JBW, et al. A brief measure for assessing generalized anxiety disorder: the GAD-7. Arch Intern Med 2006;166:1092-7.

41 Kroenke K, Spitzer RL, Williams JB. The PHQ-9: validity of a brief depression severity measure. J Gen Intern Med 2001;16:606-13.

42 Spitzer RL, Kroenke K, Williams JB. Validation and utility of a self-report version of PRIME-MD: the PHQ primary care study. primary care evaluation of mental disorders. patient health questionnaire. JAMA 1999;282:1737-44

43 Bender AM, Lawson D, Werthner P. et al. The clinical validation of the athlete sleep screening questionnaire: an instrument to identify athletes that need further sleep assessment. Sports Med Open 2018:4:23.

44 Driller MW, Mah CD, Halson SL. Development of the athlete sleep behavior questionnaire: a tool for identifying maladaptive sleep practices in elite athletes. Sleep Sci 2018:11:37-44

45 Samuels C, James L, Lawson D, et al. The athlete sleep screening questionnaire: a new tool for assessing and managing sleep in elite athletes. Br J Sports Med 2016:50:418-22.

46 Bradley KA, DeBenedetti AF, Volk RJ, et al. AUDIT-C as a brief screen for alcohol misuse in primary care. Alcohol Clin Exp Res 2007;31:1208-17.

47 Dawson DA, Grant BF, Stinson FS, et al. Effectiveness of the derived alcohol use disorders identification test (AUDIT-C) in screening for alcohol use disorders and risk drinking in the US general population. Alcohol Clin Exp Res 2005:29:844-54.

48 de Meneses-Gaya C, Zuardi AW, Loureiro SR, et al. Alcohol use disorders identification test (audit): an updated systematic review of psychometric properties. Psychol Neurosci 2009;2:83-97.

49 Brown RL, Rounds LA. Conjoint screening questionnaires for alcohol and other drug abuse: criterion validity in a primary care practice. Wis Med J 1995;94:135-40.

50 Brown RL, Leonard T, Saunders LA, et al. The prevalence and detection of substance use disorders among inpatients ages 18 to 49: an opportunity for prevention. Prev Med 1998;27:101-10. 
51 Couwenbergh C, Van Der Gaag RJ, Koeter M, et al. Screening for substance abuse among adolescents validity of the CAGE-AID in youth mental health care. Subst Use Misuse 2009;44:823-34.

52 Dyson V, Appleby L, Altman E, et al. Efficiency and validity of commonly used substance abuse screening instruments in public psychiatric patients. J Addict Dis 1998;17:57-76

53 Meersseman P, Vanhoutte S, Van Damme J, et al. A comparative study of screening instruments and biomarkers for the detection of cannabis use. Subst Abus 2016;37:176-80.

54 Sattagornpornprom N, Wannasewok K, Bussaratid S, et al. Validity of CAGE-AID in screening for amphetamine dependence or abuse in pregnancy. J Psychiatr Assoc Thailand 2013;58:385-94.

55 Martinsen M, Holme I, Pensgaard AM, et al. The development of the brief eating disorder in athletes questionnaire. Med Sci Sports Exerc 2014;46:1666-75.

56 Kessler RC, Adler L, Ames M, et al. The world Health organization adult ADHD selfreport scale (ASRS): a short screening scale for use in the general population. Psychol Med 2005:35:245-56.

57 Ustun B, Adler LA, Rudin C, et al. The world Health organization adult attentiondeficit/hyperactivity disorder self-report screening scale for DSM-5. JAMA Psychiatry 2017;74:520-6.

58 Dodd S, Williams LJ, Jacka FN, et al. Reliability of the mood disorder questionnaire: comparison with the structured clinical interview for the DSM-IV-TR in a population sample. Aust N Z J Psychiatry 2009;43:526-30.
59 Hirschfeld RM, Williams JB, Spitzer RL, et al. Development and validation of a screening instrument for bipolar spectrum disorder: the mood disorder questionnaire. Am J Psychiatry 2000;157:1873-5.

60 Hirschfeld RMA, Calabrese JR, Weissman MM, et al. Screening for bipolar disorder in the community. J Clin Psychiatry 2003;64:53-9.

61 Prins A, Ouimette P, Kimerling R, et al. The primary care PTSD screen (PC-PTSD): development and operating characteristics. Primary Care Psychiatry 2004;9:9-14.

62 Spoont MR, Williams JW, Kehle-Forbes S, et al. Does this patient have posttraumatic stress disorder?: rational clinical examination systematic review. JAMA 2015;314:501-10

63 Holtgraves T. Evaluating the problem gambling severity index. J Gamb/ Stud 2009:25:105-20.

64 Sharp C, Steinberg L, Yaroslavsky I, et al. An item response theory analysis of the problem gambling severity index. Assessment 2012;19:167-75.

65 Loewy RL, Bearden CE, Johnson JK, et al. The prodromal questionnaire (PQ): preliminary validation of a self-report screening measure for prodromal and psychotic syndromes. Schizophr Res 2005;77:117-25.

66 Ising HK, Veling W, Loewy RL, et al. The validity of the 16-item version of the prodromal questionnaire (PQ-16) to screen for ultra high risk of developing psychosis in the general help-seeking population. Schizophr Bull 2012:38:1288-96. 